\title{
Review of: "Genome-wide association study followed by trans-ancestry meta-analysis identify 17 new risk loci for schizophrenia"
}

Changqing Sun

Potential competing interests: The author(s) declared that no potential competing interests exist.

Overall speaking, this is a comprehensive study of schizophrenia which includes GWAS in Chinese population, meta-analysis by combing the current results with previous GWASs conducted in EAS and EUR, then followed by post-GWAS analysis.

By combing the GWAS in Chinese with the previous GWASs, it showed improved prefiction performance in predicting the case/control status of GWAS in Chineses only.

Furthermore, the current study identified 17 novel risk loci for schizophrenia, which highlights the importance and necessity of conducting genetic study in different populations. These findings not only provide new insights into genetic etiology of

schizophrenia, but also facilitate to delineate the pathophysiology of schizophrenia and develop new therapeutic targets. 